\title{
NEURAL NETWORK-BASED IDENTIFICATION AND MPC CONTROL OF SMB CHROMATOGRAPHY
}

\author{
Chaoyong Wang* Sebastian Engell * Felix Hanisch * \\ * Process Control Laboratory \\ Department of Chemical Engineering, University of Dortmund \\ D-44221 Dortmund, Germany
}

\begin{abstract}
In this contribution, the identification and control of nonlinear SMBchromatographic processes are discussed. Instead of using the physical manipulated process variables, the flow rates of extract, desorbent, and recycle, and the switching time directly, a new set of input variables ( $\beta$-factors) is employed as control inputs to reduce input/output couplings. A new measure of the front positions of the axial concentration profiles is used as outputs. Multi-layer neural network models are identified for this nonlinear MIMO system. The identified model is used in a model predictive control algorithm. In this algorithm a parameter varying linear model is employed which avoids the on-line computation of the nonlinear optimization problem. The simulation results show that the identified model gives a very good approximation of the process models and the LPVMPC scheme has a good control performance.
\end{abstract}

\section{Keywords: Simulated-Moving-Bed (SMB), chromatography, neural networks, nonlinear identification, model predictive control (MPC), linear parameter varying models.}

\section{INTRODUCTION}

Simulated Moving Bed (SMB) chromatography (Ruthven and Ching, 1989) is an important separation process in industrial production, especially of finechemical and pharmaceutical products, where difficult separation tasks often occur. In recent years, a lot of research concentrated on modelling, simulation and optimization of the SMB process. A rigorous model of the SMB process leads to a description by partial differential equations, which can only be solved numerically with high computational effort. It is difficult to control the SMB process on-line using such process models directly. Therefore, a black-box model is needed for the use in on-line control.

Recently, some control strategies of the SMB process were discussed. Several choices of the input and output variables for process control were proposed (Kloppenburg and Gilles, 1999), e.g. the product purities as controlled variables and external flow rates as inputs. For controller design, the process model was used. Identification and control of SMBprocesses with linear isotherm were discussed in (Dünnebier et al., 2000; Klatt et al., 2000). To regulate the process around the optimal trajectory, an IMC controller was designed based upon linear models of the ARX type. In (Wang et al., 2001) neural network based model identification was discussed for a nonlinear SMB process. In this study, a new set of output variables is defined to simplify the model structure. The neural network based identification and the control strategy based on these models are discussed for a nonlinear SMB process. The remainder of the paper is organized as follows: In Section 2, a brief description of the operation of SMB processes is given and the related identification and control problems are formulated. In Section 3, the neural network based identification of the process is discussed. In Section 4, the LPVMPC controller based on the NN model will be described. The identified models and the control strategy are evaluated by simulations, finishing with some conclusions in the final section.

\section{PROBLEM STATEMENT}

\subsection{Brief description of the SMB process}

A typical SMB system is shown in Fig. 1. All columns are connected in series by pumps and multi-way, multi-position solenoid valves. The system has two input ports, through which the feed mixture $(A+B)$ and the desorbing solvent $D$ can continuously be injected into the system. There are also two withdrawal ports, from which the separated pure products extract $A$ and raffinate $B$ are withdrawn. The cascade of columns is divided into four sections or zones. During the operation, the four connecting points, desorbent, extract, feed and raffinate, are shifted after each specified period (called as the switching time $\tau$ ) by one column in the fluid flow direction. Due to the different adsorption affinities of the two components in the packed columns, an extract product $(A)$ is collected at the extract port and a raffinate product $(B)$ is withdrawn 
from the raffinate port. If the process parameters are appropriately chosen for a given SMB equipment and a given separation task, a cyclic-steady-state (CSS), in which the process undergoes an identical transient during each switching period, is reached after a startup period.

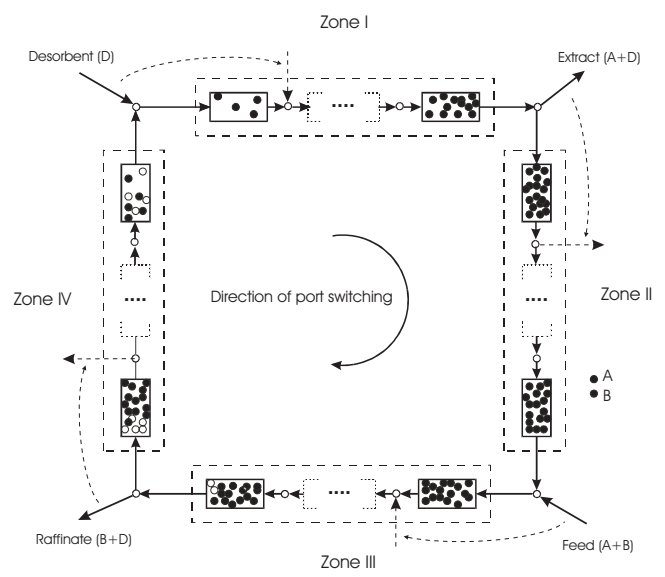

Figure 1. Schematic diagram of a SMB process

The SMB process can be described rigorously by a set of partial differential and algebraic equations (Dünnebier and Klatt, 1999). The dynamics of a single column in a SMB process are described by the general rate model in Eq. (1):

$$
\begin{aligned}
& \frac{\partial c_{i}}{\partial t}+\frac{6(1-\epsilon) k_{l, i}}{\epsilon d_{p}}\left(c_{i}-\left.c_{p i}\right|_{r=0.5 d_{p}}\right) \\
&=D_{a x, i} \frac{\partial^{2} c_{i}}{\partial x^{2}}-u \frac{\partial c_{i}}{\partial x} \\
&\left(1-\epsilon_{p}\right) \frac{\partial q_{i}}{\partial t}+\epsilon_{p} \frac{\partial c_{p, i}}{\partial t}=\epsilon_{p} D_{p, i}\left[\frac{1}{r^{2}} \frac{\partial}{\partial r}\left(r^{2} \frac{\partial c_{p, i}}{\partial r}\right)\right]
\end{aligned}
$$

The model is completed by the mass balances at the nodes and boundary and initial conditions which represent the switching process. This rigorous model is employed here for the generation of the identification data and for simulation of the controlled system.

A two-level control architecture was proposed in (Klatt et al., 2000) for the control of the SMB process. On the upper level, the optimal operating trajectory is calculated off-line by dynamic optimization based on the rigorous process model. On the lower level, on-line process control is performed to keep the process on the optimal trajectory in case of disturbances, plant/model mismatch and measurement noise. Here a simplified model which is identified from simulated data of the rigorous process model is needed. The identification procedure and the design of the on-line controller will be covered in the following sections.

\subsection{Choice of input and output variables for control}

In this study, the same inputs are used as in (Wang et al., 2001): We assume that the feed flow rate $Q_{F}$ and the feed concentrations $c_{f e e d A, B}$ are constant during the separation in order to maintain a high productivity, and the extract flow rate $Q_{E}$, the desorbent flow rate $Q_{D}$, the recycle flow $Q_{R}$ and the switching time $\tau$ are used as the manipulated variables of the process. To reduce the strong interactions of the process, the socalled $\beta$-factors, $\beta_{1, \ldots, 4}$, are used as the new system input variables for identification and control, which were introduced for the computation of optimal operating policies of the SMB-process. They are defined by a nonlinear input transformation (Dünnebier and Klatt, 1999):

$$
\begin{gathered}
Q_{S}=\frac{Q_{F}}{\frac{\partial g_{A}}{\partial c_{A}} / \beta_{3}-\frac{\partial g_{B}}{\partial c_{B}} \beta_{2}}=\frac{(1-\epsilon) A_{c o l} L}{\tau} \\
Q_{E}=Q_{S}\left(\frac{\partial g_{A}}{\partial c_{A}} \beta_{1}-\frac{\partial g_{B}}{\partial c_{B}} \beta_{2}\right) \\
Q_{D}=Q_{S}\left(\frac{\partial g_{A}}{\partial c_{A}} \beta_{1}-\frac{\partial g_{B}}{\partial c_{B}} / \beta_{4}\right) \\
Q_{4}=Q_{S}\left(\frac{\partial g_{B}}{\partial c_{B}} / \beta_{4}+\frac{\epsilon}{1-\epsilon}\right) \\
g_{i}=\epsilon_{p} c_{i}+\left(1-\epsilon_{p}\right) q_{i}\left(c_{A}, c_{B}\right), \quad(i=A, B)
\end{gathered}
$$

where $q_{i}(.,$.$) are the adsorption isotherm functions, c_{i}$ are the fluid phase concentrations.

The new system input variables $u_{i}$ are:

$$
\mathbf{u} \triangleq\left[u_{1}, \cdots, u_{4}\right]^{T} \triangleq\left[\beta_{1}, \cdots, \beta_{4}\right]^{T} .
$$

The axial profile at the end of a switching period in CSS is an important characteristic curve of the separation process and represents the separation behavior. The purpose of on-line control is to keep the adsorption and desorption fronts at their optimal positions. In (Wang et al., 2001) characteristic points of the front curves of the axial profiles were used as system output variables and a related neural network model was identified. But this choice of the outputs requires the fronts to be approximately of Gaussian form, and the structure of the obtained model is still complex. In this study, a new set of outputs $\left(y_{1}, y_{2}, y_{3}\right.$ and $\left.y_{4}\right)$ is defined as shown in Fig. 2. Consider the nominal axial concentration profiles $\left(c_{a}^{(0)}(x), c_{b}^{(0)}(x)\right)$. Deviations from this desired profile result in different shapes and positions of the observed profiles $\left(c_{a}^{(k)}(x), c_{b}^{(k)}(x)\right)$ at instance $k$. These deviations can be quantified by the areas between nominal and current profiles. To reduce the influence of strong deformations of the front shapes, only the regions between $5 \%$ and $60 \%$ percent of the nominal plateau heights are considered, resulting in the four shaded areas in Fig. 2. These areas describe profile changes sufficiently well and are sensitive to the front shifts. But their computations are not so sensitive to the deformation of the profiles as the outputs used in (Wang et al., 2001; Dünnebier et $a l ., 2000)$. Furthermore, with this choice of the outputs, the structure of the obtained model is simple. 


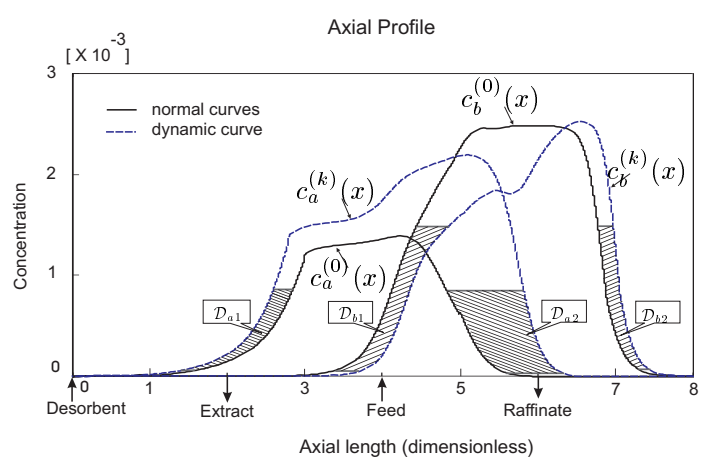

Figure 2. Definition of output variables. Solid line: CSS profile, dashed line: dynamic profile at the $k$-th period.

Formally,

$$
\begin{array}{ll}
y_{1}(k)=\iint_{\mathcal{D}_{a 1}} d c d x, & y_{3}(k)=\iint_{\mathcal{D}_{a 2}} d c d x \\
y_{2}(k)=\iint_{\mathcal{D}_{b 1}} d c d x, & y_{4}(k)=\iint_{\mathcal{D}_{b 2}} d c d x .
\end{array}
$$

Because the real axial concentration profiles cannot be measured on-line, the Assembled Elution Profile (AEP) (Dünnebier et al., 2000), which is assembled from measurements of the elution profiles at the ends of the columns during a switching period is used as an approximation of the axial profile. In this work, we assume that all elution profiles from the individual columns are measurable and the AEP profiles are used for identification and control.

Since the values of the inputs are held constant during each switching period, the switching time is regarded as the discrete sampling time of the system, and the SMB process is then regarded as a discrete time MIMO system with four inputs and four outputs. The identification and the control of such a discrete time MIMO $(4 \times 4)$ system is the main focus of this study.

\section{NEURAL NETWORK BASED IDENTIFICATION OF PROCESS MODELS}

The identification method discussed in (Wang et al., 2001 ) is used here for SMB processes with the above new definition of the output variables. The identification procedure will be outlined in the following paragraphs. A typical laboratory scale nonlinear SMB process for the separation of two enantiomers is used as an example in the study. The process parameters and the operating point are taken from (Strube et al., 1998) and also given in (Wang et al., 2001).

\subsection{Analysis of the process dynamics}

The step responses of the process in Fig. 3 show that the process is strongly nonlinear and not completely decoupled, even though its interactions have been largely reduced by using $\beta$-factors as inputs. From these curves, some rough structural information about the dynamics of the process can also be extracted for an initial guess of the model structure. For example, the inputs which have a strong influence on one output can be determined, so that the model only contains those inputs and/or other outputs as model inputs that have a strong effect on this output.
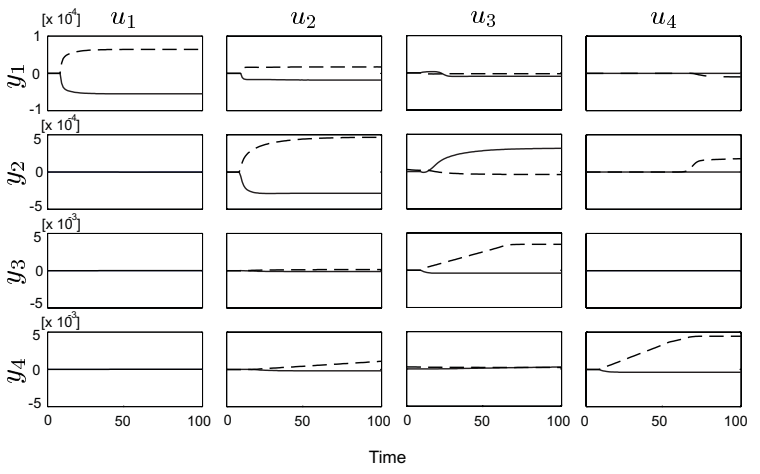

Figure 3. Step responses. Solid line: $\delta \beta_{i}=3 \% \beta_{i 0}$, dashed line: $\delta \beta_{i}=-3 \% \beta_{i 0}$

Multilayer perceptron feedforward neural networks (NARX model) are then used as process models for the problem because of the high dimension of the input space of the system. To reduce the computational effort, four $(4 \times 1)$-MISO sub-models denoted as $M_{1}$, $M_{2}, M_{3}$ and $M_{4}$ are identified separately for each output. The $i$-th MISO NARX model $M_{i}$ is represented by the following equation:

$$
\begin{aligned}
y_{i}(k+1)= & \Phi_{i}\left[y_{i}(k), \ldots, y_{i}\left(k-n_{y_{i}}\right), y_{j}\left(k-d_{i, y_{j}}\right),\right. \\
& \ldots, y_{j}\left(k-d_{i, y_{j}}-n_{i, y_{j}}\right), u_{l}\left(k-d_{i, l}\right), \\
& \left.\ldots, u_{l}\left(k-d_{i, l}-n_{i, l}\right)\right] \\
= & \Phi_{i}(\varphi(k)) \\
\varphi(k) \triangleq & {\left[\cdots, y_{j}\left(k-o_{1}\right), \cdots, u_{l}\left(k-o_{2}\right), \cdots\right]^{T} } \\
& j, l=1 \ldots 4, \quad j \neq i .
\end{aligned}
$$

where $\Phi_{i}$ is the parametrized nonlinear function described by a MLP neural network with a hidden layer, $\boldsymbol{\varphi}(k)$ is the regressor, $d_{i, y_{j}}, d_{i, l}$ are time delays, and $n_{y_{i}}, n_{i, y_{j}}, n_{i, l}$ are the numbers of lags of the past outputs and inputs, respectively.

The neural network MISO NARX models were trained using the Levenberg-Marquardt optimization method.

\subsection{Exciting signal}

To obtain a set of informative experimental data for nonlinear identification, a random uniform sequence (RUS) (Wang et al., 2001) was used as an exciting input signal. The simulation experiments were conducted under MATLAB based on the general-rate model in Eq.(1) of the SMB process. A large amount of data was collected as a training set (data length $\mathrm{N}=$ 6500 ) for the identification, so that the trained model is not over-estimated and the model structure can be 
adjusted correctly according to the gradient information. Another independent data set with a length of 500 samples was generated as a test data set for the validation of the identified models.

\subsection{Determination of the structural parameters of the model}

The structural parameters of the model cannot be accurately determined in advance. The gradient method (Schultz, 1998; Wang et al., 2001) was used to determine the model orders. After training of the NN model, the average gradients of the output with respect to the input terms $\bar{g}_{i}=\frac{1}{N} \sum_{k=1}^{N}\left|\frac{\partial y(k+1)}{\partial \varphi_{i}(k)}\right|$ are calculated using the identified models. The average gradient distribution $\bar{g}_{i}$ is then used to adjust the model structure by adding more important terms to the model and/or removing less important terms from the model.

- If $\bar{g}_{o_{j, 1}}^{(i)}=\frac{1}{N} \sum_{k}\left|\frac{\partial y_{i}(k+1)}{\partial u_{j}(k-d+1)}\right|<\delta$ (a small real number) for all integers $0 \leq d<d_{j 0}$ and $\bar{g}_{o_{j, 1}}^{(i)}>\delta$ for $d=d_{j 0}+1$, then the integer $d_{j 0}$ is regarded as the time delay for the input variable $u_{j}$;

- If $\bar{g}_{o_{j, 2}}^{(i)}=\frac{1}{N} \sum_{k}\left|\frac{\partial y_{i}(k+1)}{\partial u_{j}\left(k-d_{j 0}+l+1\right)}\right|>\delta$ for $l=$ $n_{j 0} \geq 1$ and $\bar{g}_{o_{j, 2}}^{(i)}<\delta$ for $l>n_{j 0}$, then the integer $n_{j 0}$ is chosen as the lag number for $u_{j}$.

In this way, we modified the structural parameters for retraining of the model and finally determined all structural parameters. In addition, another important structural parameter $n_{H}$, the number of the neurons in the hidden layer of the neural networks, was determined by the SVD (Singular Value Decomposition) method (Sentoni et al., 1996).

\subsection{Identification results}

The four MISO neural network-based models were identified separately using the training data. The obtained model structures are given in Table 1.

Table 1. Model Structures

\begin{tabular}{ccccc}
\hline \multicolumn{5}{c}{ Models } \\
& $M_{1}$ & $M_{2}$ & $M_{3}$ & $M_{4}$ \\
\hline$\underline{\mathbf{u}}$ & $\left\{u_{1}, u_{2}, u_{3}\right\}$ & $\left\{u_{2}, u_{3}\right\}$ & $\left\{u_{2}, u_{3}\right\}$ & $\left\{u_{2}, u_{4}\right\}$ \\
$n_{y i}$ & 3 & 3 & 3 & 4 \\
$n_{i}$ & $\{3,2,2\}$ & $\{3,2\}$ & $\{2,3\}$ & $\{2,4\}$ \\
$d_{i}$ & $\{1,1,2\}$ & $\{1,1\}$ & $\{1,1\}$ & $\{1,1\}$ \\
$n_{H}$ & -- & 6 & 3 & 6 \\
\hline
\end{tabular}

$n_{H}$ : the neuron number in the hidden layer of $\mathrm{NN}$.

$\underline{\mathbf{u}}$ : input terms of the model.

An example of the multi-step ahead predictions, and the pure predictions of the identified NN models on the validation data are displayed in Fig. 4. The predictions of the NN models are compared with the results of linear ARX models in Table 2. It can be seen that some submodels (e.g. $M_{1}$ and $M_{2}$ ) can be accurately represented by linear models while $M_{3}$ and $M_{4}$ can be much improved using NN model. The NN models have high accuracy and show a good long range prediction performance.

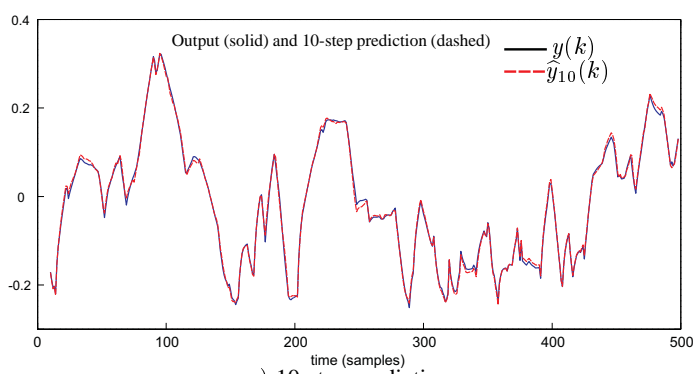

a) 10-step prediction

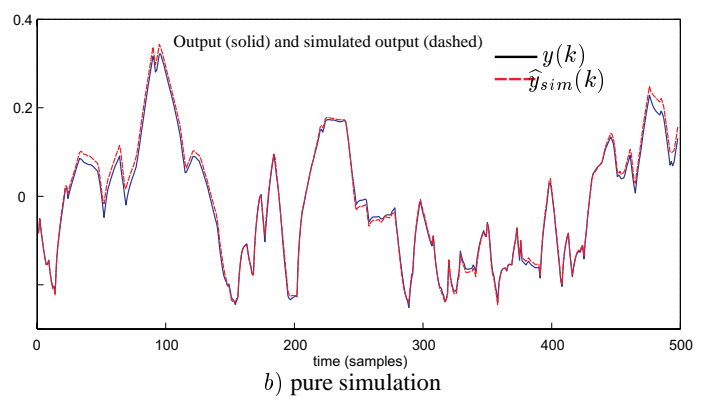

Figure 4. a) 10-step ahead prediction and $b$ ) pure prediction of $M_{3}$

Table 2. Prediction errors of the models

\begin{tabular}{cllll}
\hline Models & Types & \multicolumn{3}{c}{ Pred. Errors (RMSE) } \\
\cline { 3 - 5 } & & 1 -step & 10 -step & simu. \\
\hline$M_{1}$ & linear arx & $1.1 \mathrm{e}-3$ & $2.4 \mathrm{e}-3$ & $2.58 \mathrm{e}-3$ \\
& nn narx & \multicolumn{3}{c}{ not identified } \\
\hline$M_{2}$ & linear arx & $6.7 \mathrm{e}-3$ & $2.68 \mathrm{e}-2$ & $2.7 \mathrm{e}-2$ \\
& nn narx & $1.9 \mathrm{e}-3$ & $1.4 \mathrm{e}-2$ & $1.95 \mathrm{e}-2$ \\
\hline$M_{3}$ & linear arx & $1.04 \mathrm{e}-2$ & $4.03 \mathrm{e}-2$ & $5.86 \mathrm{e}-2$ \\
& nn narx & $3 \mathrm{e}-3$ & $6.3 \mathrm{e}-3$ & $1.25 \mathrm{e}-2$ \\
\hline$M_{4}$ & linear arx & $2.09 \mathrm{e}-2$ & $7.32 \mathrm{e}-2$ & 0.29 \\
& nn arx & $3 \mathrm{e}-3$ & $1.9 \mathrm{e}-2$ & $4.9 \mathrm{e}-2$ \\
\hline
\end{tabular}

Note: 1. predictions are based on validation data. 2. nn narx: neural network nonlinear ARX model.

Because of the limited amount of the experimental training data, the obtained model has still some kind of uncertainty, although it has passed the validation test. As a final indirect proof of the effectiveness of the identified model, it is applied to control design. This will be discussed in the next section.

\section{LINEAR PARAMETER VARYING MODEL PREDICTIVE CONTROL (LPVMPC) BASED ON THE NN MODEL}

\subsection{The LPVMPC algorithm}

If we linearize the $\mathrm{NN}$ model, we can obtain a linearized ARX model at each time instance $k$. Its parameters vary with the actual process state, as shown in Fig. 5. This figure demonstrates that the parameters 
of the linearized model change strongly at the different process states. This means that the system dynamics can also change strongly during the operation.

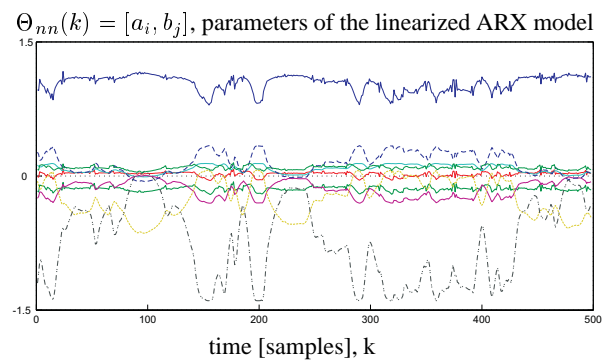

Figure 5. Variations of the parameters of the linearized model obtained from the NN model $M_{3}$

For such a system a linear MPC scheme can not achieve a satisfactory result. On the other hand, the solution of linear MPC problems is simple and the commercial software is available (e.g. MATLAB MPC Toolbox) for linear systems. In contrast, nonlinear MPC is not easily applied because of the difficulty of the nonlinear optimization problem. Therefore, the linear parameter varying model (Arkun et al., 1998) is extended and a linear parameter varying MPC scheme (LPVMPC) is introduced for the process which is shown in Fig 6.

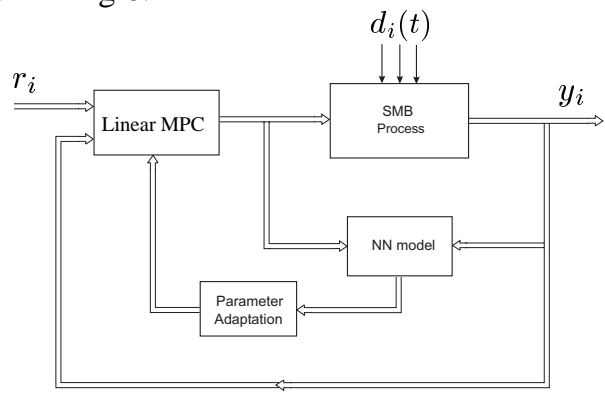

Figure 6. Linear Parameter Varying MPC control system structure

In Fig. 6, the linear MPC (LMPC) controller and the nonlinear NN model are the two basic parts. At each sampling instance the parameters of the controller are based on the NN model and the new on-line measurements, and then the new control is computed by the standard LMPC algorithm.

A general linear MPC controller is obtained by (Morari and Ricker, 1995):

$$
\begin{aligned}
\min _{\{u(p)\}} \mathbf{J}_{\mathbf{k}}= & \sum_{p=k+1}^{k+M_{p}} \widehat{\mathbf{e}}_{p}^{T} \mathbf{Q} \widehat{\mathbf{e}}_{p}+\sum_{p=k+1}^{k+M_{c}} \Delta \mathbf{u}_{p}^{T} \mathbf{R} \Delta \mathbf{u}_{p} \\
\text { s.t. } \quad & \mathbf{X}(k+1)=\mathbf{F} \cdot \mathbf{X}(k)+\mathbf{G} \cdot \mathbf{u}(k) \\
& \mathbf{Y}(k+1)=\mathbf{H} \cdot \mathbf{X}(k) \\
& U_{\min , i} \leq u_{i}(k+p) \leq U_{\max , i} \\
& \left|\triangle u_{i}(k+p)\right| \leq \triangle U_{\max } \\
& Y_{\min , i} \leq y_{i}(k+p) \leq Y_{\max , i}
\end{aligned}
$$

where $\widehat{\mathbf{e}}(k+p) \triangleq \mathbf{r}(k+p)-\mathbf{Y}(k+p \mid k), \mathbf{r}(k+p)$ is the reference sequence, $\mathbf{F}, \mathbf{G}, \mathbf{H}$ are system ma- trices, $U_{\min , i}, U_{\max , i}$ and $\Delta U_{\max }$ are control limits, $Y_{\min , i}, Y_{\max , i}$ are output limits, $\mathbf{Q}, \mathbf{R}, M_{p}, M_{c}$ are weighting matrices and control parameters, respectively.

For LPVMPC the following models are used:

a) A linear model around the set point:

$$
\begin{gathered}
\mathbf{Y}(k+1)=\mathbf{Y}_{0}+\boldsymbol{\Theta}_{0} \cdot\left[\boldsymbol{\varphi}(k)-\boldsymbol{\varphi}_{0}\right] \\
\mathbf{Y} \triangleq\left[y_{1}, \cdots, y_{4}\right]^{T}
\end{gathered}
$$

where $\Theta_{0}$ is the parameter matrix of the linear model.

b) A linearized model at time instance $k$ is obtained by linearization of the NN model of Eq. (5) as follows:

$$
\mathbf{Y}(k+1)=\mathbf{Y}_{k}+\Theta_{n n}(k) \cdot[\varphi(k)-\varphi(k-1)]
$$

where the $\Theta_{n n}(k)$ is the parameter matrix of the linearized model.

c) The new weighted linear model at time instance $k$ for LPVMPC is updated by

$$
\begin{aligned}
\mathbf{Y}(k+1) & =\mathbf{Y}_{0}+\boldsymbol{\Theta}_{n e w}(k) \cdot\left[\boldsymbol{\varphi}(k)-\boldsymbol{\varphi}_{0}\right] \\
\boldsymbol{\Theta}_{\text {new }}(k) & =\mathbf{K}_{c o m p} \boldsymbol{\Theta}_{n n}(k)+\left(\mathbf{I}-\mathbf{K}_{c o m p}\right) \boldsymbol{\Theta}_{0} \\
\mathbf{K}_{c o m p} & \triangleq \operatorname{diag}\left(k_{c 1}, k_{c 2}, \cdots, k_{c 4}\right)
\end{aligned}
$$

where $\mathbf{K}_{c o m p}$ is the compensation matrix and $k_{c i}$ is the compensation factor for each control loop, taking a value in $[0,1]$.

According to Eq.(7), (8) and (9), the model used for LPVMPC is updated at each instance $k$. This control strategy, which uses the linear MPC result and the NN model, can improve the control performance without having to solve a general nonlinear optimization problem, comparing with LMPC.

\subsection{Simulation Results}

The identified NN model and the LPVMPC are used for control of a nonlinear SMB process. The control system is simulated to reject the disturbances in $\beta_{i}$ and in feed concentrations $c_{\text {feed }}$. The simulation shows that the LPVMPC system can reject the disturbance significantly compared to the linear MPC system. Two simulation cases are shown in Fig. 7, 8.

In these figures the output responses of LPVMPC system are improved significantly comparing with LMPC, but the inputs of the two systems in both cases are quite similar. The reason for this is that both channels $\left(u_{3} \rightarrow y_{3}\right.$ and $\left.u_{4} \rightarrow y_{4}\right)$ contain integration dynamics, as indicated in their step responses in Fig. 3. As a result, a small change in input $\left(u_{3}\right.$ or $\left.u_{4}\right)$ will result in significant change in output $\left(y_{3}\right.$ or $\left.y_{4}\right)$.

In addition, the LMPC system based on the linear model of the SMB process is stable. But its control responses are quite slow. In LPVMPC the nonlinear dynamics of the process are considered and this 
compensation of the model nonlinearity improves the control performance. The compensation factor $k_{c i}$ in Eq.(9) has a large effect on the system performance and stability. A larger value of $k_{c i}$ means a strong influence of the nonlinear dynamics on the system and will result in faster system responses. If it is too large, the LPVMPC system will be unstable. Conversely, the system with a smaller $k_{c i}$ is stable, but quite slow. An appropriate value of $k_{c i}$ should be carefully chosen by simulation.
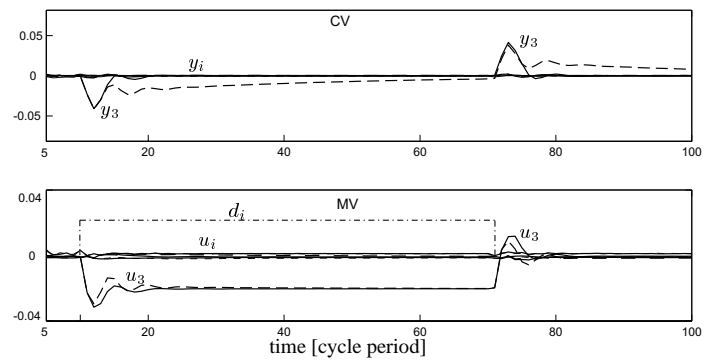

Figure 7. LPVMPC and LMPC in presence of disturbance $d_{i}=\delta \beta_{3}=0.02 \beta_{30}$. Solid line: LPVMPC, dashed line: LMPC
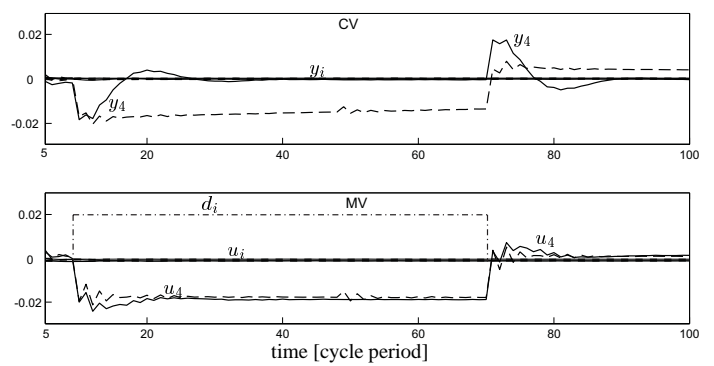

Figure 8. LPVMPC and LMPC in presence of disturbance $d_{i}=\delta \beta_{4}=0.02 \beta_{40}$. Solid line: LPVMPC, dashed line: LMPC

\section{CONCLUSIONS}

In this paper we have investigated the neural networkbased identification of a nonlinear SMB-process. The $\beta$-factors were used as inputs, and the front area variations of the AEP-profiles were defined as outputs. Since the process is sampled after each switching time $\tau$, a nonlinear discrete time $(4 \times 4)$-MIMO system is obtained. Multi-layer perceptron (MLP) networks with one hidden layer were employed to identify the process model. The identified models were evaluated both on training data and on validation data by comparing the multi-step-ahead prediction and the pure prediction. The results have shown that the identified models exhibit satisfactory long range prediction performance, which is favorable for a model based control application. In addition, it has also been shown that the identified model is simpler than that with the characteristic points as system outputs (Wang et al., 2001). Furthermore, a linear parameter varying MPC based on the NN model is proposed and applied successfully to the control of a nonlinear SMB process. This LPVMPC scheme exploits the linear MPC technique, providing linear parameter varying models from the NN models. Thus the nonlinear optimization problem can be avoided and the available MPC tools can be used. Simulations show a large improvement of the control performance compared to linear MPC for an enatiomer separation using SMB chromatography.

\section{REFERENCES}

Arkun, Y., A. Banerjee and N. Lakshmanan (1998). Self scheduling MPC using LPV models. In: Nonlinear Model Based Control (R. Berber and C. Kravaris, Eds.). Kluwer Academic Publishers.

Dünnebier, G. and K.U. Klatt (1999). Optimal operation of simulated moving bed chromatographic processes. Comp. Chem. Engng. 23, 189-192.

Dünnebier, G., S. Engell, K.-U. Klatt and M. Turnu (2000). Reduced dynamic models of simulated moving bed chromatographic processes. In: Proceedings of ADCHEM 2000, Pisa, Italy. pp. 201206.

Klatt, K.-U., F. Hanisch, G. Dünnebier and S. Engell (2000). Model-based optimization and control of chromatographic processes. Comp. Chem. Eng. 24, 1119-1126.

Kloppenburg, E. and E.D. Gilles (1999). Automatic control of the simulated moving bed process for c8 aromatics separation using asymptotically exact Input/Output-linearization. J. Process Control 9, 41-50.

Morari, M. and N.L. Ricker (1995). Matlab Model Predictive Control Toolbox User's Guide. The MathWorks, Inc.

Ruthven, D.M. and C.B Ching (1989). Countercurrent and simulated counter-current adsorption separation processes. Chem. Eng. Sci. 44, 10111038.

Schultz, J. (1998). Identifikation nichtlinearer dynamischer Systeme mit künstlichen neuronalen Netzen. Dr. -Ing. Dissertation. Universität Karlsruhe, Germany.

Sentoni, G.B., O. Agamennoni, A. Desages and J. Romagnoli (1996). Approximate models for nonlinear process control. AIChE J. 42(8), 2240-2250.

Strube, J., S. Haumreisser and H. Schmidt-Traub (1998). Comparison of batch elution and continuos simulated moving bed chromatography. Organic Process Research and Development 2, 305-319.

Wang, C., K.-U. Klatt, G. Dünnebier, S. Engell and F. Hanisch (2001). Neural network-based identification of the SMB Chromatographic process. In: Preprints of 6th IFAC Symposium on DYCOPS, 2001, Cheju, Korea. pp. 183-188. 